\title{
In Vivo Investigation of Filter order Influence in Eigen-based Clutter Filtering for Color Flow Imaging
}

\author{
Lasse Lovstakken*, Alfred C. H. Yu ${ }^{\dagger}$, and Hans Torp* \\ *Department of Circulation and Medical Imaging, Norwegian University of Science and Technology, Trondheim, Norway \\ ${ }^{\dagger}$ Department of Electrical and Electronic Engineering, University of Hong Kong, Pokfulam, Hong Kong SAR \\ Correspondance email: lasse.lovstakken@ntnu.no, alfred.yu@eee.hku.hk
}

\begin{abstract}
Eigen-based adaptive filters have shown potential for providing a superior attenuation of clutter in color flow imaging. Critical for the success of this technique is the correct selection of filter order. In this work we review and compare filter order selection schemes for eigen-based filters in an in vivo context.

Data was acquired from a thyroid tumor $(P R F=250 \mathrm{~Hz}$, ensemble size $=12$, where substantial tissue movement was present due to carotid artery pulsations, respiratory movements, and probe navigation. Eigen-filtering performance was evaluated for 1) an eigenvalue spectrum threshold, 2) a threshold on the ratio of successive eigenvalues, and 3) a threshold on eigenvector mean frequency estimated by the autocorrelation approach.

Based on the observed eigenvalue and eigen-frequency distributions in analytical and in vivo examples, all filter order algorithms investigated suffered from potential pitfalls in specific Doppler scenarios. In the in vivo examples, the fixed order eigenfilter gave a sufficient suppression of clutter, but also removed substantial blood signal. Thresholding the ratio of eigenvalues better retained signal from blood, but also spurious artifacts was observed. The most consistent results were achieved by thresholding the mean frequency of the eigenvectors. The results demonstrate that given a suitable filter order algorithm, robust filtering can be achieved with the eigen-based approach.
\end{abstract}

\section{INTRODUCTION}

Central to conventional color flow imaging (CFI) algorithms is the use of a clutter filter to attenuate signal from surrounding tissue structures. This clutter component is present also in vessel lumens due to reverberations and beam sidelobes, and can have a substantially higher signal power than the blood signal component of interest. If not sufficiently attenuated, clutter may lead to a false coloring of tissue regions and biased blood velocity estimates.

Clutter rejection has conventionally been done by high-pass filtering the slow-time signal ensembles at each range bin [1]. However, due to the inability of these filters to adapt their stop band to the clutter component, a sufficient attenuation may not be achieved when there are substantial tissue movements that give rise to nonzero Doppler frequencies.

More advanced filters have been proposed that do adapt the stop band to the clutter component [2]. One particular method that has shown promise is the eigen-based approach. In this approach the slow-time signal is expanded into its principal signal components as given by the eigenvectors of the signal correlation matrix, and clutter filtering is achieved by subtracting the principal signal components identified as clutter from the original signal [2], [3]. However, some aspects of this technique still remains to be investigated before it can be established in CFI. One important aspect is the selection of filter order, i.e. the number of clutter eigen-components to subtract from the original signal. If the filter order is chosen incorrectly, clutter may not be sufficiently attenuated, or blood signal may also be removed in the filtering process. Further, since the Doppler signal mixture vary throughout the image region and the cardiac cycle, the most suitable filter order vary accordingly, thus an adaptive filter order algorithm is needed.

In this work we investigate strategies for filter order selection in eigen-based clutter filtering in CFI, and compare the performance of these algorithms in an in vivo example where adaptive clutter filtering may be of particular use.

\section{BACKGROUND THEORY}

\section{A. General signal and filtering model}

The Doppler signal from each image sample is in general assumed to consist of three components; clutter $c$ present due to tissue signal reverberations and beam side lobes, blood signal $b$, and thermal noise $n$. Assuming independent components, the general signal model is then given by:

$$
s=c+b+n .
$$

To separate the tissue and blood components, the signal is expanded into its principal components as can be achieved through the Karhunen-Loève expansion, given by [4]:

$$
\boldsymbol{x}=\sum_{k=1}^{N_{D}} \gamma_{k} \boldsymbol{e}_{k}, \quad E\left\{\gamma_{k} \gamma_{l}^{*}\right\}= \begin{cases}\lambda_{k} & k=l \\ 0 & k \neq l\end{cases}
$$

where $N_{D}$ is the ensemble size, and $e_{k}$ and $\lambda_{k}$ are the eigenvectors and corresponding eigenvalues of the signal correlation matrix $\mathbf{R}_{x}$, sorted on decreasing eigenvalues. Each eigenvector, or principal component, accounts for a part of the total signal variation, and can be associated with any of the three constituent signal components in (1). In particular for the clutter filtering algorithm, eigen-components representing clutter are identified and subtracted from the original slowtime signal.

\section{B. Overview of eigen-based clutter filtering}

After data acquisition, an ensemble of $N_{D}$ slow-time samples is available for each image point, sampled at the pulse repetition frequency (PRF). The first step in eigen-based clutter filtering is the estimation of the slow-time signal correlation 


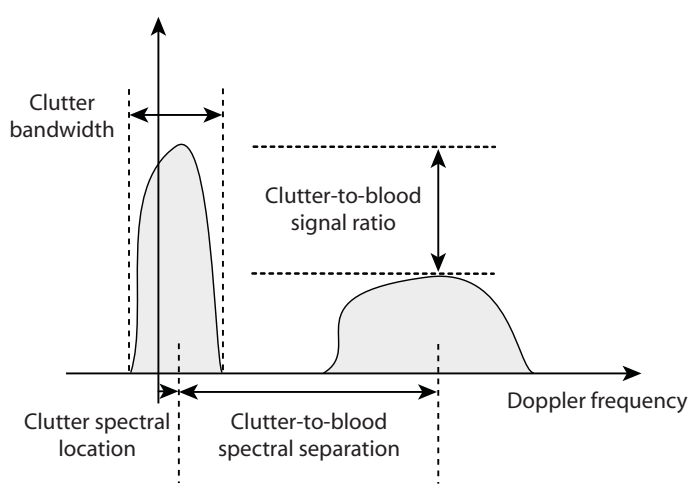

Fig. 1. An illustration of signal characteristics that influence filter order selection for a hypothetical Doppler spectrum. Important parameters include the clutter bandwidth $B_{c}$ and spectral location $w_{c}$, and the clutter-to-blood signal ratio $\mathrm{CBR}$ and spectral separation CBSS.

matrix. In this work the correlation matrix is estimated by averaging the outer product of $M$ signal vectors in a spatial region centered around each image point, as given by

$$
\hat{\mathbf{R}}_{x}=\frac{1}{M} \sum_{m=1}^{M} \boldsymbol{x}_{m} \boldsymbol{x}_{m}^{* T},
$$

where the averaging of signal vectors $\boldsymbol{x}_{m}$ is done both radially and laterally. In the second step, $N_{D}$ eigenvectors and eigenvalues of $\hat{\mathbf{R}}_{x}$ are computed via algorithms such as power iterations or QR factorization [4]. A subset of these eigenvectors $\boldsymbol{\Phi}_{c}$ is in a third step identified as representing clutter, and is in the fourth and final step removed through projection filtering $\boldsymbol{y}=\mathbf{P} \boldsymbol{x}$. The filter projection matrix is here formed as:

$$
\mathbf{P}=\mathbf{I}-\sum_{k=1}^{K_{c}} \boldsymbol{e}_{k} \boldsymbol{e}_{k}^{* T}, \quad \boldsymbol{e}_{k} \in \boldsymbol{\Phi}_{c}
$$

where $K_{c}$ is the number of clutter eigen-components.

\section{Filter Order Selection}

In order to successfully attenuate the clutter signal component using the eigen-based approach, it is necessary to identify 1) the number of eigen-components $K_{c}$ that represent clutter, and 2) the particular subset of clutter eigen-components $\boldsymbol{\Phi}_{c}$. The order of the resulting clutter filter is defined as $K_{c}-1$.

The distribution of eigen-components over the constituent signal components will be signal dependent. From a Fourier perspective one can identify several parameters that will influence the selection of clutter eigen-components. Some are shown in Fig. 1 for a hypothetical Doppler spectrum, including the clutter-to-blood signal ratio (CBR), the clutter Doppler center frequency $\omega_{c}$ and bandwidth $B_{c}$, and the clutter-toblood spectral separation (CBSS). These parameters will vary for different imaging scenarios, and also during the cardiac cycle. For high-frequency imaging CBR may actually approach zero due to the Rayleigh scattering properties of blood. The most dominant eigen-components may then be distributed over both clutter and blood. Further, for a narrow CBSS, a larger overlap between components in the eigenspectrum can be seen.
An increasing clutter bandwidth typically relates to an increasing number of clutter eigen-components $K_{c}$. This bandwidth is mostly given by the observation time as determined by the PRF and ensemble size $N_{D}$, but also through accelerated movement. The clutter Doppler component may also exhibit a shift in center frequency due to tissue movement.

Algorithms for selecting clutter eigen-components in CFI has previously been reported based on the information available in both the eigenvalue spectrum [2], [3], [5], and in the frequency content of individual eigenvectors [6]. These concepts and their potential pitfalls will be further described in the following subsections.

\section{A. Eigenvalue-based algorithms}

The eigenvalues contain information about the signal energy represented by the individual eigenvectors. A dominant signal component that constitute a large part of the total signal energy will be represented by eigenvectors with large corresponding eigenvalues. Due to its dominant and low-bandwidth nature this is often the case for the clutter component [2]. This observation has been the foundation for selection criteria used in prior investigations, where a subset of the most dominant eigenvectors have been selected as clutter eigencomponents [2], [3].

One proposed algorithm has been to include a fixed number of the most dominant eigenvectors. However, as the signal characteristics vary in both space and time, an improved algorithm might be to select the eigenvectors with corresponding eigenvalues above a predefined threshold $\lambda_{c}$. The subset of eigenvectors is then given by:

$$
\boldsymbol{\Phi}_{c}=\left\{\boldsymbol{e}_{k} \mid \lambda_{k}>\lambda_{c}\right\}, \quad k=1, \ldots, N_{D} .
$$

The threshold $\lambda_{c}$ can for instance be set relative to the most dominant eigenvalue.

Similary, the number of clutter eigen-components can also be chosen based on the shape of the eigenspectrum, more specifically by thresholding the eigenvalue differences $\lambda_{k}-$ $\lambda_{k+1}$ or ratios $\lambda_{k} / \lambda_{k+1}$ [5]. The rationale for such algorithms is that the eigenspectrum will flatten once higher bandwidth signals such as that of blood and thermal noise are introduced.

\section{B. Frequency-based algorithms}

By investigating the frequency content of the individual eigenvectors, information is given about the spectral band of the Doppler spectrum the eigenvectors represent. As the tissue movement typically is small compared to that of blood flow, it is reasonable to identify eigenvectors representing lowfrequency parts of the Doppler spectrum as clutter.

The mean frequency of the k'th eigenvector $\boldsymbol{e}_{k}$, can be estimated using the autocorrelation technique [7], as given by:

$$
\hat{\omega}_{k}=\angle \hat{R}_{1, k}=\angle\left[\frac{1}{N_{D}-1} \sum_{m=0}^{N_{D}-2} \boldsymbol{e}_{k}^{*}(m) \boldsymbol{e}_{k}(m+1)\right],
$$

where $\hat{R}_{1, k}$ is the sample correlation function of lag one, and $\hat{\omega}_{k}$ is the estimated mean frequency of eigenvector $\boldsymbol{e}_{k}$. 

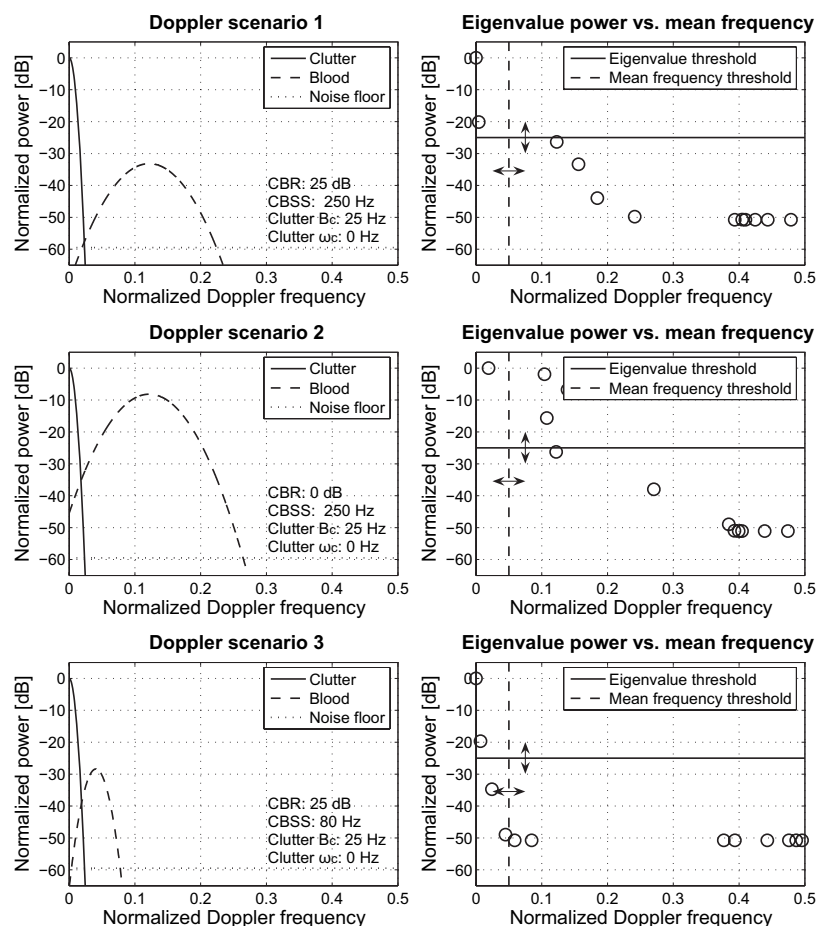

Fig. 2. The concepts and pitfalls of different filter order algorithms. In the first example, a moderate signal scenario is shown where both methods are shown to identify the correct clutter eigen-components. In the second example, the occurrence of zero CBR can be seen to infer problems for the eigenvaluebased algorithm. In the third row, a narrow CBSS infers problems for the frequency-based algorithm.

An eigenvector is included in the clutter basis subset if its mean frequency lies within a predefined clutter bandwidth $B_{c}$ centered around the center clutter frequency $\omega_{c}$, as given by:

$$
\boldsymbol{\Phi}_{c}=\left\{\boldsymbol{e}_{k}|| \hat{\omega}_{k}-\omega_{c} \mid<\frac{1}{2} B_{c}\right\}, \quad k=1, \ldots, N_{D} .
$$

In practise, the mean Doppler clutter frequency $\omega_{c}$ can often be assumed zero. However, as reported by Yu et al [6], an improved performance can be achieved by setting it equal to the mean frequency of the most dominant eigenvector from (6), $\hat{\omega}_{c}=\hat{\omega}_{1}$, as this may compensate for mean frequency shifts of the clutter spectrum.

\section{Comparison of filter order algorithms}

The concepts and potential pitfalls of eigenvalue and frequency thresholding are illustrated in Fig. 2 for three analytical Doppler scenarios generated using the stationary Doppler signal model described in [8]. The different Doppler scenarios and relevant parameters related to Fig. 1 are shown in the left column. In the right column the eigenvalues of the signal correlation matrix are plotted against the mean frequency of the corresponding eigenvectors. This plot allows us to compare the performance of the selection criteria, and also to investigate if a combination of the criteria can be used to improve the identification of clutter eigen-components.

In the first Doppler scenario a moderate CBR and CBSS is given, and it can be observed that both thresholding cri-
TABLE I

ULTRASOUND ACQUISITION PARAMETERS

\begin{tabular}{cc}
\hline Acquisition parameter & Value \\
\hline \hline Clinical object & Thyroid nodule \\
Probe & GE M12L \\
Center frequency, $f_{0}$ & $6.7 \mathrm{MHz}$ \\
No. of periods in pulse & 2.5 \\
F-number (transmit / receive) & $2.5 / 1.4$ \\
Pulse repetition frequency (PRF) & $250 \mathrm{~Hz}$ \\
Ensemble size & 12 \\
\hline
\end{tabular}

teria can give reasonable results, isolating the dominant and low-frequency clutter component. A potential pitfall for the eigenvalue-based criteria is illustrated for the second scenario, where a zero CBR is given. In this case it can be observed that it is difficult to choose an eigenvalue threshold without also including blood eigen-components. The frequency-based algorithm can in this case still isolate the clutter eigencomponents. In the third row an example is shown where a narrow CBSS is given. The resulting eigenvalues from clutter and blood can then be seen to cluster in frequency, causing problems for the frequency-based thresholds. As can be seen for this example, the eigenvalue threshold is then still able to isolate the clutter eigen-components.

\section{EXPERIMENTAL RESULTS}

The filter order algorithms were evaluated in the in vivo setting of a thyroid tumor. The aim in diagnosis might here be to investigate the vascularization inside the tumor, which may be related to malignancy. Tissue movement was present due to carotid artery pulsations, respiratory movements, and probe navigation. Coupled with a low PRF of $250 \mathrm{~Hz}$ to image the lowest possible flow made this a challenging scenario for clutter filters. Relevant ultrasound acquisition parameters are listed in Table I. The filter parameter setup, e.g. the threshold value, were chosen to produce the best visual result for the given examples. It was apparently not possible to achieve reasonable results using a simple eigenvalue threshold for these data, and these results are therefore not shown.

In Fig. 3 and 4, power-Doppler images for two frames of thyroid data are shown for four different filters. A) A 5th order polynomial regression filter was used as a reference. In the same image, the thyroid tumor and carotid artery have been outlined. As can be seen, this filter did not attenuate clutter sufficiently for the given examples. B) An eigenfilter with a fixed order of 4 gave a sufficient attenuation of clutter, but also severely attenuated the blood signal. C) Thresholding the eigenvalue ratios did retain the signal from blood, but spurious artifacts could also be observed as shown in Fig. 4. D) Using a mean-frequency threshold provided consistent clutter attenuation while also retaining the blood signal.

The rightmost column in Fig. 3 and 4, shows the distribution of eigenvalues vs. mean frequency as introduced in Fig 2. The examples are taken from a region of tissue only, and a region with tissue and blood. Observe for instance how the mean frequency algorithm most probably also includes a blood eigencomponent in the lower plot of Fig 3, and 

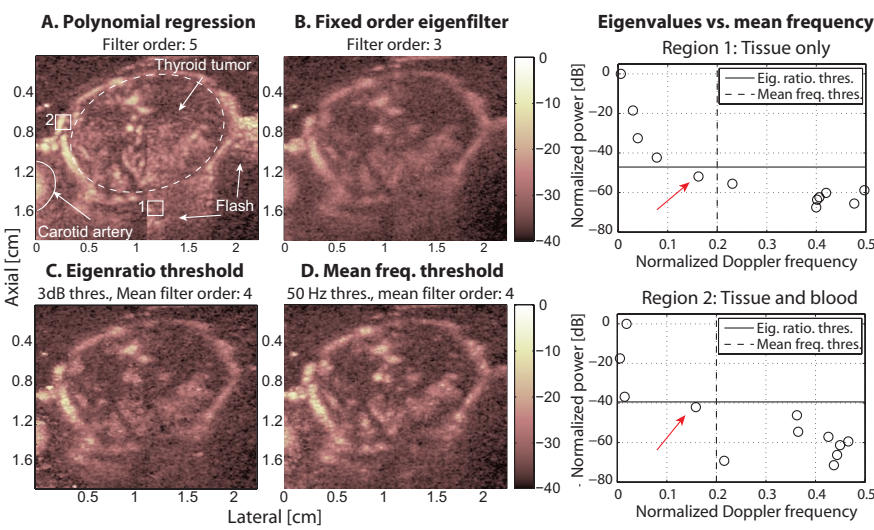

Fig. 3. Example 1: Filtered images of a Thyroid tumor. A) Polynomial regression, 5th order B) Fixed order Eigenfilter, 4th order, C) Thresholding ratio of eigenvalues, D)Thresholding mean frequency of eigenvectors. In the rightmost column, eigenvalue vs. frequency plots provide examples of regions where both thresholding methods fail for the given example.
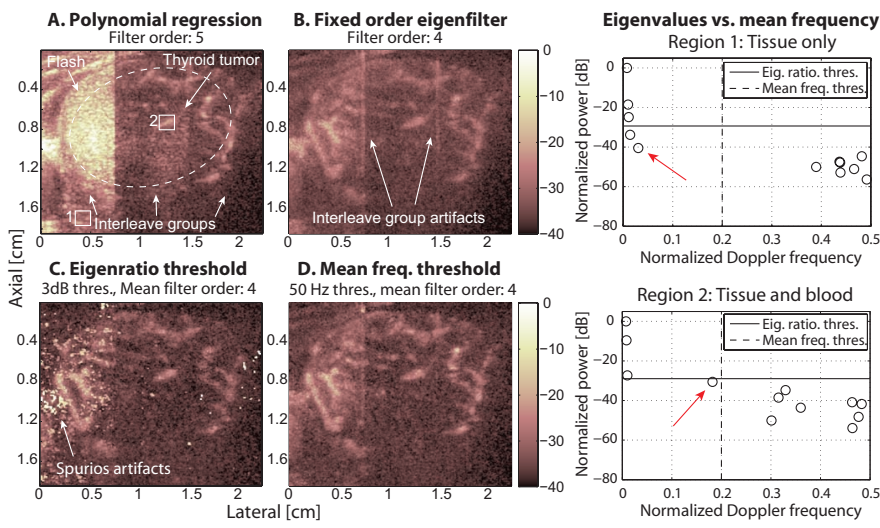

Fig. 4. Example 2: Filtered images of a Thyroid tumor. A) Polynomial regression, 5th order B) Fixed order Eigenfilter, 4th order, C) Thresholding ratio of eigenvalues, D)Thresholding mean frequency of eigenvectors. In the rightmost column, eigenvalue vs. frequency plots provide examples of regions where both thresholding methods fail for the given example.

how the eigenratio algorithm does not include all clutter eigencomponents in the upper plot in Fig. 4.

\section{DISCUSSION}

Different algorithms for the selection of filter order in eigenbased filtering and their potential pitfalls have been reviewed through analytical Doppler spectra and in vivo examples. Theoretically, both the eigenvalue and frequency-based methods may lead to suboptimal results when used exclusively.

Common to eigenvalue-based algorithms is the assumption of a dominant clutter component. This is however not always the case, especially for higher imaging frequencies due to the Rayleigh scattering properties of blood. A disadvantage of the frequency-based algorithm is the predefined clutter bandwidth threshold. As the bandwidth varies in both space and time, the threshold should vary accordingly. Also, the frequency content covered by the eigenvectors may be distributed over extended regions, representing both clutter and blood. For these cases the estimated mean frequency can be severely biased.
Observing that one algorithm successfully identifies the correct clutter components while another fails and vice versa, indicates that the combination of the criteria could be used to achieve a more robust filter selection algorithm. Referring to the eigenvalue vs. frequency plots in Fig. 2, a 2-D segmentation algorithm could be developed to more properly isolate the eigen-components for the wide range of Doppler scenarios that may be encountered. This is considered future work.

For the in vivo examples, the most consistent results were achieved when using the mean frequency criteria. Although the eigenratio algorithm also provided reasonable results, spurious artifacts was observed which needs further investigation. The results demonstrate that given a suitable filter order selection algorithm, robust filtering performance can be achieved with the eigen-based approach. More work is needed to map the performance of the filter order selection algorithms and eigenbased clutter filtering in a wider range of clinical applications.

\section{CONCLUSION}

All algorithms for filter order selection investigated suffered from pitfalls in certain Doppler signal scenarios. In in vivo examples, the mean frequency criteria gave the most consistent results, and demonstrated that robust filtering can be achieved even in a challenging low-PRF example with tissue movement. By combining the information available in both the eigenvalues and eigenvectors, an improved performance may be achieved. This is the focus for further work.

\section{ACKNOWLEDGMENTS}

The authors would like to thank Kristin Helset and SveinErik Masoy at Trondheim University Hospital, Norway, for providing the in vivo data used in this investigation. The authors are also grateful to Paul Y.S. Cheung (HKU) and Richard S.C. Cobbold (UT) for their support.

\section{REFERENCES}

[1] S. Bjærum, H. Torp, and K. Kristoffersen, "Clutter filter design for ultrasound color flow imaging," IEEE Trans. Ultrason. Ferroelec. Freq. Contr., vol. 49, pp. 204-216, 2002.

[2] S. Bjærum, H. Torp, and K. Kristoffersen, "Clutter filters adapted to tissue motion in ultrasound color flow imaging," IEEE Trans. Ultrason. Ferroelec. Freq. Contr., vol. 49, pp. 693-704, 2002.

[3] D. E. Kruse and K. W. Ferrara, "A new high resolution color flow system using an eigendecomposition-based adaptive filter for clutter rejection,' IEEE Trans. Ultrason. Ferroelec. Freq. Contr., vol. 49, pp. 1384-1399, 2002.

[4] T. K. Moon and W. C. Stirling, Mathematical Methods and Algorithms for Signal Processing. Upper Saddle River, USA: Prentice Hall Inc., 2000.

[5] L. Løvstakken, S. Bjærum, K. Kristoffersen, R. Haaverstad, and H. Torp, "Real-time adaptive clutter rejection filtering in color flow imaging using power method iterations," IEEE Trans. Ultrason. Ferroelec. Freq. Contr., vol. 53, pp. 1597-1608, 2006.

[6] A. C. H. Yu and R. S. C. Cobbold, "Single-ensemble-based eigenprocessing methods for color flow imaging-Part I. the Hankel-SVD filter," IEEE Trans. Ultrason. Ferroelec. Freq. Contr., vol. submitted, 2007.

[7] C. Kasai, K. Namekawa, A. Koyano, and R. Omoto, "Real-time twodimensional blood flow imaging using an autocorrelation technique," IEEE Trans. Sonics Ultrason., vol. 32, pp. 458-464, 1985.

[8] H. Torp, K. Kristoffersen, and B. Angelsen, "Autocorrelation technique in color flow imaging, signal model and statistical properties of the autocorrelation estimates," IEEE Trans. Ultrason., Ferroelec., Freq. Contr., vol. 41, pp. 604-612, 1994. 